\title{
Characteristics of Progressive Systemic Sclerosis in a Cohort of Egyptian Patients
}

\section{Mahmoud $A^{1 *}$, Alhefny $A^{1}$, Abugabal $\mathbf{M}^{1}$, Abdelmoteleb $\mathbf{S}^{1}$, Alhassanein $\mathrm{KF}^{1}$, Abdelzaher $\mathbf{A}^{1}$, Sayed $\mathrm{S}^{2}$, Haroon $\mathrm{MM}^{2}$ and Soliman $\mathrm{H}^{3}$}

${ }^{1}$ Division of Rheumatology, Department of Internal Medicine, Ain Shams University' Cairo, Egypt

${ }^{2}$ Department of Rheumatology, Faculty of Medicine, Cairo University, Egypt

${ }^{3}$ Department of Rheumatology, Maaday Military Hospital, Cairo, Egypt

*Corresponding author: Adel Mahmoud, Division of Rheumatology, Department of Internal Medicine, Ain Shams University, Cairo, Egypt, Tel: 01005265425, 002-02-22585015; E-mail: dr_adel_mahmoud25@yahoo.com

Received date: February 07, 2018; Accepted date: February 08, 2018; Published date: February 14, 2018

Citation: Mahmoud A, Alhefny A, Abugabal M, Abdelmoteleb S, Alhassanein KF, et al (2018) Characteristics of Progressive Systemic Sclerosis in a Cohort of Egyptian Patients. Arch Med Vol No:10 Iss No:1:7

Copyright: (C) 2018 Mahmoud A, et al. This is an open-access article distributed under the terms of the Creative Commons Attribution License, which permits unrestricted use, distribution, and reproduction in any medium, provided the original author and source are credited.

\section{Abstract}

Objective: To estimate the frequency of epidemiological, clinical and laboratory characteristics of progressive systemic sclerosis in a cohort of Egyptian patients.

Methods: Fifty systemic sclerosis patients were included. These patients were subjected to detailed history taking, clinical and rheumatological examination, Measuring the dermal skin thickness by the modified Rodnan skin score (mRSS), Nail fold capillaroscpy (NFC) and the relevant radiological, laboratory and immunological investigations.

Results: Our results revealed that the mean age at time of diagnosis was $32.66 \pm 13.08$ with the disease durations range from 1 to 40 years with a median of five years. Male to female ratio of 1: 5.2 and $20 \%$ of patients were smokers. Skin tightness was present in all patients, the mRSS ranges from 4 to 45 with a mean of $17.48 \pm 10.44$. ANA was detected in $98 \%$, RF was detected $4 \%$, antitopoisomerase I (antitopo I) was detected in $36 \%$ and ACA was detected in $8 \%$ of patients. $96 \%$ of patient had abnormal NFC. There were statistical significant negative correlations between $\mathrm{mRSS}$ and both of FEV ${ }_{1} \%$ and FVC\%.

There was also a statistical significant positive correlation between $\mathrm{mRSS}$ and $\mathrm{FEV}_{1} / \mathrm{FVC}$.

Conclusion: This study has shown that almost our Egyptian SSC patients have ANA seropositivity, abnormal pulmonary function tests and abnormal nailfold capillaroscopy (NFC). The study revealed that anti topo I antibody seropositivity, ILD, abnormal pulmonary function tests, worsening skin score, late pattern of NFC are more common in Diffuse Cutaneous Systemic Sclerosis (DCSSC) than Limited Cutaneous Systemic Sclerosis (LCSSC).

Also ILD in SSC patients is commonly associated with antitopo I antibody seropositivity, abnormal pulmonary function tests, worsening skin score and late pattern of NFC. Therefore ANA, antitopo I, high resolution CT chest, pulmonary function test, mRSS and NFC should be considered for early diagnosis and follow up of SSC patients.

Keywords: Systemic sclerosis; Modified rodnan skin score; Nailfold capillaroscopy; Diffuse cutaneous systemic sclerosis; Limited cutaneous systemic sclerosis

\section{Introduction}

Systemic sclerosis (SSC) is a rare multisystemic connective tissue disease characterised by microvascular damage, fibrosis of the skin and internal organs and specific immunologic abnormalities. The clinical recognisable disease is classified on the basis of extent of skin involvement into subsets with diffuse cutaneous involvement (DCSSC) and limited cutaneous involvement (LCSSC) [1].

Systemic sclerosis (scleroderma, SSC) is an autoimmune disease in which fibrosis of the skin and internal organs occur in association with small vessel vasculopathy and autoantibody production. Organ-specific and non-organ specific impairments lead to a spectrum of mild to severe limitations in physical, work and social activities, ultimately influencing health-related quality of life [2].

As with many other autoimmune disorders, scleroderma is approximately 4-5 times more common in women than men. The average age at the time of diagnosis is approximately 50 years [3].

There are 2 primary clinical subsets of the disease: limited cutaneous and diffuse cutaneous SSC. Limited cutaneous SSC is typically manifested by Raynaud's phenomenon, involvement of acral skin (hands, forearms, face, legs, and feet), gastroesophageal reflux symptoms, and, occasionally, pulmonary hypertension with or without interstitial lung disease (ILD). Nailfold capillaroscopy demonstrates dilated capillary loops, usually without capillary dropout [4]. 
Diffuse cutaneous SSc typically causes widespread and often early involvement of internal organs. Raynaud's phenomenon, proximal and/or truncal as well as acral skin involvement, arthritis, tendon friction rubs, myositis, ILD, malignant hypertension, diffuse gastrointestinal (GI) disease, and/or myocardial involvement may occur. Nailfold capillaroscopy demonstrates capillary dilatation and destruction [4].

However, not all patients with systemic sclerosis fall clearly into one of these two disease subsets, and some can change their subset assignment over time. Furthermore, some individuals present with hallmark clinical and serological features of systemic sclerosis in the absence of detectable skin involvement (systemic sclerosis sine scleroderma); others manifest features of another connective tissue disease, such as rheumatoid arthritis or polymyositis, in overlap with systemic sclerosis (overlap syndrome) [5].

As in other connective tissue disorders such as systemic lupus erythematosus, ethnicity has a role in systemic sclerosis [6].

A striking feature of systemic sclerosis is its patient-to-patient variability, and heterogeneity has been observed in clinical manifestations, autoantibody profiles, tempo of disease progression, response to treatment and survival. On the basis of the extent of their skin involvement, patients are grouped into limited cutaneous systemic sclerosis (LCSSC) and diffuse cutaneous systemic sclerosis (DCSSC) subsets [5].

The aim of this study was to estimate the frequency of epidemiological, clinical and laboratory characteristics of progressive systemic sclerosis (PSS) in a cohort of patients from Egypt to elicit any difference from that of other ethnic group.

\section{Patients and Methods}

\section{Study design}

This is a cross-sectional cohort study in which 50 adult patients, diagnosed with progressive systemic sclerosis according to the 2013 American College of Rheumatology/ European League Against Rheumatism Systemic Sclerosis classification criteria [7], were recruited from the outpatient clinic and inpatient departments of rheumatology in Ain Shams university and military hospitals in the period between November 2014 to September 2016.

\section{Study protocol}

For all patients the followings were done:

Detailed history taking, and clinical and rheumatological examination with special emphasis on age, gender, occupation, family history, smoking, duration of disease, medications and cardiac, pulmonary, renal, gastrointestinal and neuropsychiatric manifestations.

Measuring the dermal skin thickness by the modified Rodnan skin score (mRSS) in 17 evaluated areas. These areas are the face, anterior chest, anterior abdomen, and 7 bilateral sites including the upper arm, forearm, dorsum of the hand, fingers, thigh, lower leg, and dorsum of the foot. The grading of the mRSS is as follows: $0=$ normal, $1=$ thickened skin, $2=$ thickened and unable to pinch, $3=$ thickened and unable to move [8].

Laboratory and immunological profile: A blood sample was drawn from each patient to measure: CBC,ESR,CRP, AST, complete urine analysis, serum creatinine, calculated creatinine clearance by Cockcroft-Gault Equation, $\mathrm{CrCl}=(140$-age $) \times$ weight in Kilogram $/(\mathrm{Scr} \times 72)(\times 0.85$ for females $)$ [9], RF, ANA, Antitopoisomerase I antibodies and Anticentromere antibodies.

Assessment of internal organ affection according to individual patient presentation by: Plain X-rays of the chest, High resolution CT chest (HRCT) without contrast, ECG and Echocardiography, Pulmonary function tests (PFTs), Abdominal Ultrasonography, Nail fold capillary microscopy.

\section{Ethical consideration}

The participating patients gave their written informed consent, and the study protocol was approved by the regional ethics committee at Ain Shams University and military hospitals.

\section{Statistical analysis}

Statistical analyses were performed using SPSS statistical software (version 15.0; SPSS, Chicago, IL, USA). All data was expressed as mean and standard deviation (Mean \pm SD) for quantitative and parametric data. Mode was used for quantitative non parametric (extreme values) data.

Qualitative (categorical) data was expressed as numbers and percentages. For comparative statistical analysis, Student's t test was performed for quantitative variables in two independent groups; ANOVA ( $f$ ) test was performed for quantitative variables in more than two independent groups. Mann-Whitny (Z) test was performed for non-parametric quantitative variables in two independent groups.

Kruskal-Wallis (K) test was performed for non-parametric quantitative variables in more than two independent groups. Pearson Correlation coefficient ( $r$ value) test was done to correlate between two quantitative data in the same group.

Spearman Correlation coefficient test was done to correlate between two non-parametric quantitative data in the same group. Chi square $\left(\mathrm{X}^{2}\right)$ test was done to compare qualitative (categorical) variables between two or more proportions. The significance level was calculated as $\mathrm{P}<0.05=$ significant.

\section{Results}

This study was conducted on 50 adult Egyptian patients diagnosed as progressive systemic sclerosis with a mean age $41.36 \pm 12.71$ years and a mean age at diagnosis of $32.66 \pm$ 13.08 years. Eight patients (16\%) were males and 42 patients 
(84\%) were female. Disease durations range from 1 to 40 years with a median of five years (Table 1 ).

Table 1: Demographic and clinical characteristics of patients with SSC (50).

\begin{tabular}{|c|c|c|c|}
\hline Parameter & & Number $=50$ (or mean \pm SD) & Percentage (\%) \\
\hline \multirow[b]{5}{*}{ Demographic } & Females & 42 & 84 \\
\hline & Males & 8 & 16 \\
\hline & Disease duration & 5 Years & 1 to 40 \\
\hline & Age $($ mean $\pm S D)$ & 41.36 Years & \pm 12.71 \\
\hline & Smoking & 10 & 20 \\
\hline \multirow[b]{7}{*}{ Cutaneous } & Tightness & 50 & 100 \\
\hline & Puffy fingers & 39 & 78 \\
\hline & Digital tip ulcers & 34 & 68 \\
\hline & Pitting scars & 36 & 72 \\
\hline & Telangiectasia & 37 & 74 \\
\hline & Calcinosis & 16 & 32 \\
\hline & Raynaud's & 47 & 94 \\
\hline \multirow[b]{2}{*}{ Constitutional } & Weight loss & 30 & 60 \\
\hline & Fatigue & 45 & 90 \\
\hline \multirow[b]{4}{*}{ GIT } & GERD & 42 & 84 \\
\hline & Dysphagia & 29 & 58 \\
\hline & Constipation & 14 & 28 \\
\hline & Diarrhea & 13 & 26 \\
\hline \multirow[b]{3}{*}{ Musculoskeletal } & Arthralgias & 43 & 86 \\
\hline & Muscle weakness & 39 & 78 \\
\hline & Tendon friction rub & 12 & 24 \\
\hline \multirow[b]{3}{*}{ Pulmonary } & РAH & 9 & 18 \\
\hline & ILD & 25 & 50 \\
\hline & Dyspnea & 38 & 76 \\
\hline \multirow[b]{2}{*}{ Cardiovascular } & HTN & 13 & 26 \\
\hline & Palpitation & 18 & 36 \\
\hline \multicolumn{2}{|c|}{ Renal manifestations } & 10 & 20 \\
\hline \multirow[b]{8}{*}{ Neuropsychiatric } & Neuropathic pain & 27 & 54 \\
\hline & Headache & 25 & 50 \\
\hline & Depression & 26 & 52 \\
\hline & Stroke & 1 & 2 \\
\hline & Dementia & 19 & 38 \\
\hline & Seizures & 3 & 6 \\
\hline & TIA & 7 & 14 \\
\hline & Psychosis & 1 & 2 \\
\hline
\end{tabular}


The commonest clinical presentation in our SSC patients (50) was the cutaneous lesions in $100 \%$ of cases followed by equal percentages (90\%) regarding constitutional, GIT, musculoskeletal, and neuropsychiatric manifestations, followed by pulmonary and cardiovascular manifestations in $88 \%$ and $40 \%$ of cases respectively, however, renal manifestations were the least common recorded in $20 \%$ of cases (Table 1 ). The mRSS was ranging from 4 to 45 with Mean \pm SD of $17.48 \pm 10.44$. The most common affected site is fingers (100\%), then face $(78 \%)$, hands (70\%), feet (56\%), forearms (42\%), legs (36\%), arms (30\%), thighs $(28 \%)$, chest $(26 \%)$ and the least affected site is the abdomen (20\%) (Table 2).

Table 2: Modified rodnan skin score (mRSS) in SSC patients (50).

\begin{tabular}{|c|c|}
\hline Parameter & Mean \pm SD or No. $=50(\%)$ \\
\hline $\mathrm{mRSS} / 51$ & $17.48 \pm 10.44$ \\
\hline Face & $39(78.0 \%)$ \\
\hline Arms & $15(30 \%)$ \\
\hline Forearms & $21(42.0 \%)$ \\
\hline Hands & $35(70.0 \%)$ \\
\hline Fingers & $50(100.0 \%)$ \\
\hline Chest & $13(26.0 \%)$ \\
\hline Abdomen & $10(20.0 \%)$ \\
\hline Thighs & $14(28.0 \%)$ \\
\hline Legs & $18(36.0 \%)$ \\
\hline Feet & $28(56.0 \%)$ \\
\hline
\end{tabular}

The mRSS was ranging from 4 to 45 with Mean \pm SD of $17.48 \pm$ 10.44. The most common affected site is fingers (100\%), then face $(78 \%)$, hands $(70 \%)$, feet $(56 \%)$, forearms $(42 \%)$, legs $(36 \%)$, arms $(30 \%)$, thighs $(28 \%)$, chest $(26 \%)$ and the least affected site is the abdomen (20\%) (Table 2). Sixteen patients (32\%) were presented with anemia, of them 11 patients (22\%) presented with iron deficiency anemia and 5 patients (10\%) presented with anemia of chronic illness. ANA was detected in 49 patients (98\%), RF was detected in two patients (4\%), anti-topoisomerase I was detected in 18 patients (36\%) and anti-centromere antibody (ACA) was detected in 4 patients (8\%) (Table 3 ).

Table 3: Laboratory findings in SSC patients (50).

\begin{tabular}{|c|c|}
\hline Item & Mean \pm SD or No.=50 (\%) \\
\hline RBC'S (millions/cc) & $4.25 \pm 0.56$ \\
\hline $\mathrm{Hb}(\mathrm{gm} / \mathrm{dl})$ & $11.60 \pm 1.81$ \\
\hline MCV & $78.50 \pm 7.26$ \\
\hline $\mathrm{MCH}$ & $26.21 \pm 3.79$ \\
\hline WBCS (thousands/cc) & $6.74 \pm 2.22$ \\
\hline Platelet (thousands/cc) & $256.30 \pm 90.95$ \\
\hline ESR (mm/1st hour) & 30 (21 to 45$)$ \\
\hline CRP (mg/dl) & $6(5$ to 10$)$ \\
\hline AST (u/dl) & $25.60 \pm 12.60$ \\
\hline $\operatorname{ALT}(\mathrm{u} / \mathrm{dl})$ & 17 (15 to 22$)$ \\
\hline Urea (mg/dl) & $32.64 \pm 14.27$ \\
\hline Creatinine (mg/dl) & $0.77 \pm 0.35$ \\
\hline Calculated Cr. Clearance & $120.00 \pm 40.81$ \\
\hline Albuminuria present (No./\%) & $5(10.00 \%)$ \\
\hline
\end{tabular}




\begin{tabular}{|l|l|}
\hline Granular cast present (No./\%) & $1(2.00 \%)$ \\
\hline Positive ANA (No./\%) & $49(98 \%)$ \\
\hline Positive RF (No./\%) & $2(4.0 \%)$ \\
\hline Positive Anti topo I (No./\%) & $18(36.0 \%)$ \\
\hline Positive Anticentromere (ACA) & $4(8.0 \%)$ \\
\hline $\begin{array}{l}\text { RBC: red blood corpuscles, Hb: hemoglobin, MCV: mean cell volume, MCH: mean cell hemoglobin, WBC: white blood cells, ESR: erythrocyte sedimentation rate, CRP: } \\
\text { C-reactive protein, AST:aspartate aminotransferase, ALT: alanine aminotransferase, ANA: ant nuclear antibodies, RF: rheumatoid factor, Anti topo I: anti topoisomerase I } \\
\text { antibodies. }\end{array}$ \\
\hline
\end{tabular}

Regarding to pulmonary diseases in our study, 44 patients (88\%) had abnormal PFTs, of them 42 patients (84\%) had restrictive lung disease versus 2 patients (4\%) had obstructive lung disease. 25 patients (50\%) were presented with pulmonary fibrosis detected by HRCT chest. Regarding PAH, it was recorded in 9 patients (18\%) (Tables 4-6).

Table 4: Radiological and ECG findings in SSC patients (50).

\begin{tabular}{|c|c|c|c|}
\hline Item & Result & Number $=50$ & Percentage (\%) \\
\hline \multirow[b]{3}{*}{ CT chest } & Free & 24 & $48.00 \%$ \\
\hline & Basal pulmonary fibrosis & 25 & $50.00 \%$ \\
\hline & Minimal pleural reaction & 1 & $2.00 \%$ \\
\hline \multirow[b]{5}{*}{ ECG } & Free & 43 & $86.00 \%$ \\
\hline & Hypertensive strain & 1 & $2.00 \%$ \\
\hline & PVCS & 2 & $4.00 \%$ \\
\hline & Right BBB & 1 & $2.00 \%$ \\
\hline & SVTC & 3 & $6.00 \%$ \\
\hline \multirow[b]{7}{*}{ Echocardiography } & Negative & 36 & $72.00 \%$ \\
\hline & MR & 5 & $10.00 \%$ \\
\hline & AR & 2 & $4.00 \%$ \\
\hline & PAH & 9 & $18.00 \%$ \\
\hline & $\mathrm{TR}$ & 4 & $8.00 \%$ \\
\hline & Concentric LV hypertrophy & 1 & $2.00 \%$ \\
\hline & Dilated Rt side & 1 & $2.00 \%$ \\
\hline \multirow[b]{7}{*}{ Abdominal US } & Free & 44 & $88.00 \%$ \\
\hline & Fatty liver & 1 & $2.00 \%$ \\
\hline & Grade I nephropathy & 1 & $2.00 \%$ \\
\hline & Grade III nephropathy & 1 & $2.00 \%$ \\
\hline & Hepatomegaly & 3 & $6.00 \%$ \\
\hline & Splenomegaly & 2 & $4.00 \%$ \\
\hline & Hepatosplenomegaly & 2 & $4.00 \%$ \\
\hline
\end{tabular}

Table 5: Pulmonary function tests (PFTs) in SSC patients (50).

Item

No. $=50(\%)$ or Mean \pm SD

PFT 


\begin{tabular}{|l|l|l|}
\hline \multirow{5}{*}{} & Moderate restriction & $13(26.0 \%)$ \\
\cline { 2 - 3 } & Normal & $6(12.0 \%)$ \\
\cline { 2 - 3 } & Severe obstruction & $2(4.0 \%)$ \\
\cline { 2 - 3 } & Severe restriction & $14(28.0 \%)$ \\
\hline FEV $1 \%$ & Mean \pm SD & $66.12 \pm 19.01$ \\
\hline FVC & Mean \pm SD & $63.56 \pm 22.18$ \\
\hline FEV $/$ FVC & Mean \pm SD & $108.17 \pm 22.25$ \\
\hline
\end{tabular}

Table 6: Nailfold capillaroscopic finding in SSC patients (50).

\begin{tabular}{|c|c|c|}
\hline \multicolumn{2}{|l|}{ Item } & No. $=50(\%)$ or Mean \pm SD \\
\hline \multicolumn{2}{|l|}{ Abnormal NFC } & $48(96.0 \%)$ \\
\hline \multicolumn{2}{|l|}{ No. of capillaries (Mean \pm SD) } & $6.90 \pm 1.76$ \\
\hline \multicolumn{2}{|l|}{ Enlarged cap } & $48(96.0 \%)$ \\
\hline \multicolumn{2}{|l|}{ Mega cap } & $29(58.0 \%)$ \\
\hline \multicolumn{2}{|l|}{ Hemorrhage } & $33(66.0 \%)$ \\
\hline \multicolumn{2}{|l|}{ Loss of cap } & $38(76.0 \%)$ \\
\hline \multicolumn{2}{|l|}{ Cap. Ramification } & $2(4.0 \%)$ \\
\hline \multicolumn{2}{|l|}{ Tortuous cap } & $1(2.0 \%)$ \\
\hline \multirow[b]{4}{*}{ Pattern } & Normal & $2(4.0 \%)$ \\
\hline & Early & $28(56.0 \%)$ \\
\hline & Active & $13(26.0 \%)$ \\
\hline & Late & $7(14.0 \%)$ \\
\hline \multirow[b]{5}{*}{ Staging } & 0 & $2(4.0 \%)$ \\
\hline & 1 & $12(24.0 \%)$ \\
\hline & II & $19(38.0 \%)$ \\
\hline & III & $10(20.0 \%)$ \\
\hline & IV & $7(14.0 \%)$ \\
\hline \multirow[b]{5}{*}{ Scoring } & 0 & $2(4.0 \%)$ \\
\hline & 1 & $18(36.0 \%)$ \\
\hline & 2 & $20(40.0 \%)$ \\
\hline & 3 & $9(18.0 \%)$ \\
\hline & 4 & $1(2.0 \%)$ \\
\hline
\end{tabular}

The 50 SSC Patients were classified also according to presence of ILD into 2 groups, those with ILD (25 patients) and those without ILD (25 patients), and comparative statistical analysis were done and shown in the following Table 7. The 50 SSC Patients were classified also according to presence of pulmonary hypertension (PAH) into 2 groups, those with PAH (9 patients) and those without PAH (41 patients), and comparative statistical analysis were done and shown in the following table (Tables 8 and 9).

Table 7: Comparison between SSC patients with or without ILD. 


\begin{tabular}{|c|c|c|c|c|}
\hline \multicolumn{2}{|l|}{ Cardiovascular } & $5(20.0 \%)$ & $15(60.0 \%)$ & 0.004 \\
\hline \multicolumn{2}{|l|}{ Dysphagia } & $11(44.0 \%)$ & $18(72.0 \%)$ & 0.045 \\
\hline \multicolumn{2}{|l|}{ Dementia } & $6(24.0 \%)$ & $13(52.0 \%)$ & 0.041 \\
\hline \multicolumn{2}{|c|}{ Anti-topoisomerase I positivity } & $5(20.0 \%)$ & $13(52.0 \%)$ & 0.018 \\
\hline \multicolumn{2}{|c|}{ mRSS/51(median \pm range) } & $9(4$ to 34$)$ & 20 (8 to 45$)$ & 0.002 \\
\hline \multirow{3}{*}{ PFTs(Mean \pm SD) } & $\mathrm{FEV}_{1} \%$ & $74.35 \pm 20.44$ & $57.88 \pm 13.37$ & 0.001 \\
\hline & FVC\% & $74.65 \pm 21.46$ & $52.48 \pm 16.96$ & 0 \\
\hline & $\mathrm{FEV}_{1} / \mathrm{FVC}$ & $101.72 \pm 23.49$ & $114.62 \pm 19.30$ & 0.039 \\
\hline \multirow{4}{*}{ NFC Pattern } & Normal & $0(0.0 \%)$ & $2(8.0 \%)$ & \multirow{4}{*}{0.025} \\
\hline & Early & $14(56.0 \%)$ & $14(56.0 \%)$ & \\
\hline & Active & $10(40.0 \%)$ & $3(12.0 \%)$ & \\
\hline & late & $1(4.0 \%)$ & $6(24.0 \%)$ & \\
\hline
\end{tabular}

Table 8: Comparison between SSC patients with or without PAH.

\begin{tabular}{|c|c|c|c|c|}
\hline Item & & No PAH & PAH & P-value \\
\hline Age (Mean \pm SD) & & $39.51 \pm 12.41$ & $49.78 \pm 11.09$ & 0.027 \\
\hline $\mathrm{FEV}_{1} \%($ Mean $\pm \mathrm{SD})$ & & $70.15 \pm 17.50$ & $47.75 \pm 14.82$ & 0.001 \\
\hline Renal affection & & $6(14.6 \%)$ & $4(44.4 \%)$ & 0.043 \\
\hline Dysphagia & & $21(51.2 \%)$ & $8(88.9 \%)$ & 0.038 \\
\hline Sicca complex & & $16(40.0 \%)$ & $7(77.8 \%)$ & 0.04 \\
\hline NFC hemorrhage & & $30(73.2 \%)$ & $3(33.3 \%)$ & 0.022 \\
\hline \multirow{4}{*}{ NFC Pattern } & Normal & $2(4.9 \%)$ & $0(0.0 \%)$ & \multirow{4}{*}{0.031} \\
\hline & Early & $24(58.5 \%)$ & $4(44.4 \%)$ & \\
\hline & Active & $12(29.3 \%)$ & $1(11.1 \%)$ & \\
\hline & Late & $3(7.3 \%)$ & $4(44.4 \%)$ & \\
\hline
\end{tabular}

Table 9: Correlation ( $r$ ) between mRSS and some clinical data.

\begin{tabular}{|l|l|l|}
\hline \multirow{2}{*}{ Item } & \multicolumn{2}{l|}{ MRSS/51 } \\
\cline { 2 - 3 } & P -value & 0.001 \\
\hline FEV $\%$ & -0.462 & 0.001 \\
\hline FVC\% & -0.47 & 0.035 \\
\hline FEV 1 /FVC & 0.299 & P-value \\
\hline Pulmonary affection & median (range) & 17 (6 to 45) \\
\hline ILD & 20 (10 to 45) & 0.025 \\
\hline
\end{tabular}

\section{Discussion}

Systemic sclerosis (SSC) is an orphan disease with an annual incidence of 19 per million and prevalence of 19-75 per 100,000 in the western countries. The female:male ratio is $3: 1$, and it increases in mid to late childbearing years to 8:1. Aside from varying prevalence of internal organ involvement, Raynaud's phenomenon and skin involvement are two major hallmarks clinically characterize SSC [10]. The aim of this study was to estimate the frequency of epidemiological, clinical and laboratory characteristics of progressive systemic sclerosis in a cohort of Egyptian patients to elicit any differences from that of 
other ethnic groups. In our study the patients age range from 16 to 67 years with a mean age at diagnosis of $32.66 \pm 13.08$ years. $84 \%$ of the patients were females whereas $16 \%$ were males with female to male ratio of 5.2:1. The disease durations range from 1 to 40 years with a median of five years. These findings are comparable to the results of previous large studies [11-13]. Twenty percent of the patients were smokers but we failed to find associated significant relations of smoking to either skin tightness (mRSS), raynaud's, digital tip ulcers, interstitial lung disease (ILD), pulmonary hypertension (PAH), internal organ affection, and nail fold capillaroscopy (NFC).

Similar results were obtained in 2 other studies [14,15]. On the other hand, a large Canadian study (606 SSC patients) focused on cigarette smoking and the disease manifestations of SSC [16], found that cigarette smoking had negative effects on the vascular, gastrointestinal and respiratory outcomes of SSC. Moreover, that study also demonstrated that smoking cessation actually improved vascular complications of SSC like Raynaud's phenomenon.

In our Egyptian cohort study sclerodactly was the commonest cutaneous manifestation present in $100 \%$ of patients then puffy fingers in $78 \%$, raynaud's phenomena in $94 \%$, telangiectasia in $74 \%$, pitting scars in $72 \%$, digital tip ulcers in $68 \%$, and the least was calcinosis in $32 \%$ of patients. Among the cutaneous manifestations of 46 patients from eastern India; Raynaud's phenomenon was present in $84.8 \%$ sclerodactyly in $82.6 \%$, fingertip ulceration and scarring in 63\%, telangiectasia in $23.1 \%$, puffy finger in $8.7 \%$, cutaneous calcinosis $2.2 \%$ [12]. In another Brazilian study conducted on 32 patients, skin sclerosis and Raynaud's phenomenon present in $100 \%$ of patients, digital scars in $65.6 \%$, telangiectasia in $43.7 \%$, and calcinosis in $12.5 \%$ [17]. The mean modified rodnan skin score (mRSS) in this study was $17.48 \pm 10.44$ (4 to 45 ). It showed significant positive association with pulmonary manifestations, abnormal pulmonary function tests (PFTs), and interstitial lung disease (ILD) and there was significant negative correlation between mRSS and forced vital capacity (FVC\%).

Similar results presented by Ooi and colleagues who reported that mRSS was $21.2 \pm 9.9$ ( 2 to 46 ) in a total of 45 Chinese SSC patients. They also found that $\mathrm{mRSS}$ had positive relationship with ILD and abnormal PFTs [18]. In agreement, Bhakuni et al. reported that mRSS was $15.5 \pm 8.4$ in a total of 42 Indian SSC patients and there was significant increase in the mean mRSS score for DCSSC versus LCSSC (22.1 \pm 5.6 versus $8.2 \pm 2.8)$ [19]. Regarding ILD (50\%), it was significantly associated with higher skin score, cardiac involvement, low FVC\%, and antitopoisomerase I seropositivity.

Similar findings were detected in another study [20]. Also, ILD in our patients was significantly associated with the late pattern of nialfold capillaroscopy (NFC) and the same finding was reported by by other researchers [21]. In our study $88 \%$ of patients had inadequate PFTs (The mean FVC\% was $63.56 \pm$ $22.18 \%$ ). $30 \%$ of patients had mild restriction, $26 \%$ had moderate restriction, $28 \%$ had severe restriction, and $4 \%$ had severe obstruction while $12 \%$ of patients had normal PFTs. Sumphao-Ngern et al. studied the medical records of PFTs in 249 SCC cases, of them 73 (29.3\%) patients had inadequate PFTs
(The mean FVC\% was $69.2 \pm 14.7 \%$ predicted), $52.7 \%$ had mild restrictive lung disease, $21.8 \%$ had a moderately restrictive lung disease [22]. It was reported that 40 to $75 \%$ of SSC patients have reduction in FVC, with $15 \%$ having a severe reduction (FVC $\leq$ $50 \%$ predicted) in another study [23]. Also, it was reported that $16 \%$ of 953 SSC patients presented with severe restrictive lung disease (forced vital capacity $<55 \%$ of predicted) in a different study [24].

In a Spanish study, ILD was detected in $43 \%$ of patients, the mean FVC\% was $85 \pm 22$, while FVC $<70 \%$ was detected in $25 \%$ of patients [14]. Concerning the serological finding in our study, ANAs were detected in 98\%, Anti-topoisomerase I antibodies were detected in 36\%, Anti-centromere (ACA) antibodies were detected in $8 \%$, RF was detected in $4 \%$, of patients. In a large US study (706 SSC patients), ANAs were found in $89.3 \%$, antitopoisomerase I antibodies were found in $19.6 \%(18.1 \%$ in whites and $22 \%$ in blacks) and Anti-centromere antibodies were observed in $22.1 \%$ ( $27 \%$ in whites and $9.7 \%$ in blacks) of tested patients [13].

In another study done in Malaysian rheumatology center, ANAs were positive in $83.6 \%$ and anti-Scl 70 antibodies were positive in $34.4 \%$ [25]. A different study reported that antinuclear antibodies were present in 67\%, anti-SCL-70 autoantibodies in $28 \%$ and RF in $25.9 \%$ of tested patients [26]. Tolosa-Vilella et al. observed that ANAs were found in 91\%, ACA in $45 \%$ and anti-topoisomerase antibodies in $22 \%$ of 1326 Spanish SSC patients [14]. The lower percentages of ACA prevalence in our results may be related to the darker skin color in our SSC cases and lower cases with isolated pulmonary hypertension (two cases) where ACA is commonly associated with the white races and isolated pulmonary hypertension. Also similar findings were reported that SSC Patients with combined $\mathrm{PAH}$ and ILD were diagnosed at an older age than patients with ILD alone, and had a lower incidence of ACA positivity, a higher incidence of anti-topoisomerase positivity and DCSSC when compared to SSC-PAH alone [27]. In conclusion, this study has shown that almost our Egyptian SSC patients have ANA seropositivity, abnormal pulmonary function tests and abnormal nail fold capillaroscopy (NFC). Also LCSSC is more common than DCSSC in our SSC patients. Anti-topoisomerase I (anti topo I) antibody sero-positivity, ILD, abnormal pulmonary function tests, worsening skin score, late pattern of NFC are more common in DCSSC. Also ILD in SSC patients is commonly associated with antitopo I antibody sero-positivity, abnormal pulmonary function tests, worsening skin score and late pattern of NFC. Therefore ANA, antitopo I, high resolution CT chest, pulmonary function test, mRSS and NFC should be considered for early diagnosis and follow up of SSC patients.

\section{References}

1. Smith V, Decuman S, Sulli A, Bonroy C, Piettte Y et al. (2012) Do worsening scleroderma capillaroscopic patterns predict future severe organ involvement? a pilot study. Ann Rheum Dis 71: 1636-1639.

2. Assassi S, Mayes M, Arnett F, Gourh P, Agarwal S, et al. (2010) Systemic sclerosis and lupus: Points in an interferon-mediated continuum. Arthritis Rheum 62: 589-598. 
3. Hummers L, Wigley $F(2013)$ Current diagnosis \& treatment: Rheumatology, 3rd edition: Chapter 25.

4. Furst D, Mayes M, McSweeney P, Nash R, Sullivan K, et al. (2005) Scleroderma and the scot study.

5. Allanore Y, Simms R, Distler O, Trojanowska M, Pope J, et al. (2015) Sysemic sclerosos. Nat RevDis Primers 1: 15002.

6. Gelber A, Manno R, Shah A, Woods A, Le E, et al. (2013) Race and association with disease manifestations and mortality in scleroderma: A 20-year experience at the johns hopkins scleroderma center and review of the literature. Medicine 92: 191-205.

7. Van den Hoogen F, Khanna D, Fransen J, Johnson S, Baron M, et al. (2013) 2013 Classification criteria for systemic sclerosis: An american college of rheumatology/european league against rheumatism collaborative initiative. Ann Rheum Dis 72: 1747-1755.

8. Clements $P$, Lachenbruch $p$, Siebold J, White $B$, Weiner $S$, et al. (1995) Inter and intraobserver variability of total skin thickness score (modified rodnan TSS) in systemic sclerosis. J Rheumatol 22 1281-1285.

9. Cockcroft DW, Gault MH (1976) Prediction of creatinine clearance from serum creatinine. Nephron 16: 31-41.

10. Cutolo M, Sulli A, Pizzorni C, Paolino S, Smith V (2016) Systemic sclerosis: Markers and targeted treatments. Acta Reumatol Port 41: 18-25.

11. Simeón-Aznar C, Fonollosa-Plá V, Espinosa-Garriga G, GarcíaHernández F, et al. (2012) Registry of the spanish network for systemic sclerosis: clinical pattern according to cutaneous subsets and immunological status. Semin Arthritis Rheum 41: 789-800.

12. Ghosh S, Bandyopadhyay D, Saha I, Barua J (2012): Mucocutaneous and demographic features of systemic sclerosis: A profile of 46 patients from eastern India. Indian J Dermatol 57: 201-205.

13. Mayes M, Lacey J, Beebe-Dimmer J, Gillespie B, Cooper B, et al. (2003) Prevalence, incidence, survival, and disease characteristics of systemic sclerosis in a large US population. Arthritis Rheum 48: 2246-2255.

14. Tolosa-Vilella C, Simeón-Aznar C, Colunga-Arguelles D, Rubio-Rivas M, Callejas Rubio JL, et al. (2016) Digital ulcers and cutaneous subsets of systemic sclerosis: Clinical, immunological, nailfold capillaroscopy, and survival differences in the spanish RESCLE registry. Semin Arthritis Rheum 46: 200-208.
15. Chaudhary P, Chen X, Assassi S, Gorlova O, Draeger H, et al. (2011) Cigarette smoking is not a risk factor for systemic sclerosis. Arthritis Rheum 63: 3098-3102.

16. Hudson M, Lo E, Lu Y, Hercz D, Baron M, et al. (2011) cigarette smoking in patients with systemic sclerosis. Arthritis Rheum 63: 230-238.

17. Guidolin F, Esmanhotto L, Magro C, Silva M, Skare T (2005) Prevalence of cutaneous findings in systemic sclerosis patients: Experience of a teaching hospital. An Bras Dermatol 80: 481-486.

18. Ooi G, Mok M, Tsang K, Wong $Y$, Khong $P$, et al. (2003) Interstitial lung disease in systemic sclerosis: An HRCT-clinical correlative study. Acta Radiologica 44: 258-264.

19. Bhakuni D, Vasdev V, Garg M, Narayanan K, Jain R, et al. (2012) Nailfold capillaroscopy by digital microscope in an Indian population with systemic sclerosis. Int J Rheum Dis 15: 95-101.

20. McNearney T, Reveille J, Fischbach M, Friedman A, Lisse J, et al. (2007) Pulmonary involvement in systemic sclerosis: Associations with genetic, serologic, sociodemographic, and behavioral factors. Arthritis Rheum 57: 318-326.

21. Jehangir M, Qayoom S, Jeelani S, Yousuf R (2015) Nail fold capillaroscopy in patients of systemic sclerosis and its association with disease severity as evidenced by high resolution computed tomography lung: A hospital based cross sectional study. Int J Res Med Sci 3: 3485-3489.

22. Sumphao-Ngern P, Foocharoen C, Boonsawat W, Mahakkanukrauh A, Suwannaroj S, et al. (2015) Causes and prevalence of inadequate pulmonary function testing among patients with systemic sclerosis. Arch Med Sci 11: 1255-1260.

23. Solomon J, Olson A, Fischer A, Bull T, Brown K, et al. (2013) Scleroderma lung disease. Eur Respir Rev 22: 6-19.

24. Steen V, Medsger T (2000) Severe organ involvement in systemic sclerosis with diffuse scleroderma. Arthritis Rheum 43: 2437-2444.

25. Pagalavan L, Ong S (2007) Demography, clinical and laboratory features of systemic sclerosis in a malaysian rheumatology centre. Med J Malaysia 62: 117-121.

26. Ilovi S, Oyoo G (2013) Characteristics of systemic sclerosis patients in nairobi, Kenya: A retrospective study. Afr J Rheumatol 1: 8-12.

27. Chang B, Wigley F, White B, Wise R (2003) Scleroderma patients with combined pulmonary hypertension and interstitial lung disease. J Rheumatol 30: 2398-2405. 